\title{
Fusion Driven Fission System for Space Surface Power Application
}

\author{
Terry Kammash ${ }^{1}$ and Ricky Tang ${ }^{2}$ \\ University of Michigan, Ann Arbor, MI, 48109
}

\begin{abstract}
It is well known that fission reactors are energy rich and neutron poor, while fusion reactors are neutron rich and energy poor. As a result, it may be desirable to use the neutrons produced by a fusion reactor operating at a multiplication factor $\mathbf{Q} \sim 1$ to breed fissile material in a blanket, which can also undergo fission reactions with the fast neutrons to produce power. We examine this approach in conjunction with a bi-modal fusion propulsion system which, upon reaching destination, is operated primarily as a neutron source. The neutrons produced by the DT reactions are allowed to impinge on a blanket containing thorium-232, thereby producing uranium-233. This uranium isotope has a fast neutron fission cross reaction of about 2 barns, which we employ to calculate the power density produced in a steady-state operating system. We consider a cylindrical system whose length is significantly larger than its diameter, thereby invoking a one-dimensional approximation for the neutron flux equation which we solve in conjunction with the $U^{233}$ production rate equation. For reasonable blanket dimensions consistent with the fusion plasma parameters, we calculate the power density in the fission system and find that it is about 70 times larger than would have been the case in a pure fusion reactor. Hence, such a hybrid system may be viewed as equivalent to a fusion reactor with a $\mathbf{Q} \sim 70$ that can be utilized for space surface power production with no proliferation risk.
\end{abstract}

\section{Nomenclature}

$=$ diffusion coefficient

$=$ neutron current

$=$ diffusion length

$=$ density

$=$ neutron density

$=$ plasma density

$=$ neutron flux

$=$ blanket radius

$=$ plasma radius

$=$ neutron source

$=$ absorption cross section

$=$ fission cross section

$=$ transport cross section

$=$ cross section

$=$ fusion reaction rate

\section{Introduction}

TN several previous publications (e.g. Ref. 1) we introduced a bi-modal fusion propulsion system which can be 1 used for transportation on the one hand, and for power production once destination is reached on the other. Fusion reactions in the device are initiated by fission fragments and annihilation products produced by "at rest" annihilation of antiprotons in a $\mathrm{U}^{238}$ target properly placed in the magnetic confinement chamber in which the fusion

\footnotetext{
${ }^{1}$ Stephen S. Attwood Professor Emeritus of Nuclear Engineering and Radiological Sciences, 2355 Bonisteel Blvd., Ann Arbor, MI 48109, AIAA Associate Fellow.

${ }^{2}$ Graduate Student, Dept. of Aerospace Engineering, 1320 Beal Ave., Ann Arbor, MI 48109, AIAA Member.

American Institute of Aeronautics and Astronautics 092407
} 
plasma is located. These studies have shown that very modest amounts (on the order of a few micrograms) of antiprotons are required to ignite the fusion plasma and allow the system to operate in a steady state propulsion mode that can achieve a one-way trip to Mars in about four weeks. Such a fusion system is characterized by a multiplication factor "Q" (ratio of fusion power to injected power) of slightly larger than unity, making it more readily feasible than a terrestrial power producing system that demands much larger "Q" values. The system in question can also be readily used as a power-producing system to perform tasks at the site with essentially the same amount of antiprotons and approximately the same Q value. However, the power produced is that of a typical fusion reactor, normally of low power density, thereby requiring a large plasma volume if large amounts of power are deemed necessary. This problem can be ameliorated if the neutrons from the fusion reactions are made to impinge on a blanket containing thorium-232 in order to produce the fissionable isotope uranium-233. The latter has a sizable fast neutron fission cross section, which can be utilized to produce power in the blanket and thus allowing the system to produce the large amounts of power that may be required. As will be seen shortly, such a hybrid system will have a power density that is about 70 times larger than would have been the case in a pure fusion reactor.

\section{Analysis and Results}

The fusion system of interest is the gasdynamic mirror (GDM) which is an open-ended magnetic confinement device that is designed to confine a deuterium-tritium (DT) plasma of appropriate density and temperature. We isolate a section of this cylindrical chamber and illustrate it in Fig. 1, where we employ a plasma of radius $R_{p}=5 \mathrm{~cm}$ surrounded effectively by a blanket of $R=100 \mathrm{~cm}$ radius. It is assumed that the plasma length is sufficiently large that we can treat the problem as one dimensional. The particle balance equation for the $U^{233}$ atoms is given by

$$
\frac{d N^{33}}{d t}=\varphi \sigma_{\gamma} N^{32}-\varphi \sigma_{f} N^{33}
$$

where $N^{33}$ is the $\mathrm{U}^{233}$ atom density, $N^{32}$ the thorium density, $\varphi$ the neutron flux, $\sigma_{\gamma}$ the microscopic "breeding" cross section, and $\sigma_{f}$ the fission cross section. The cross sections in

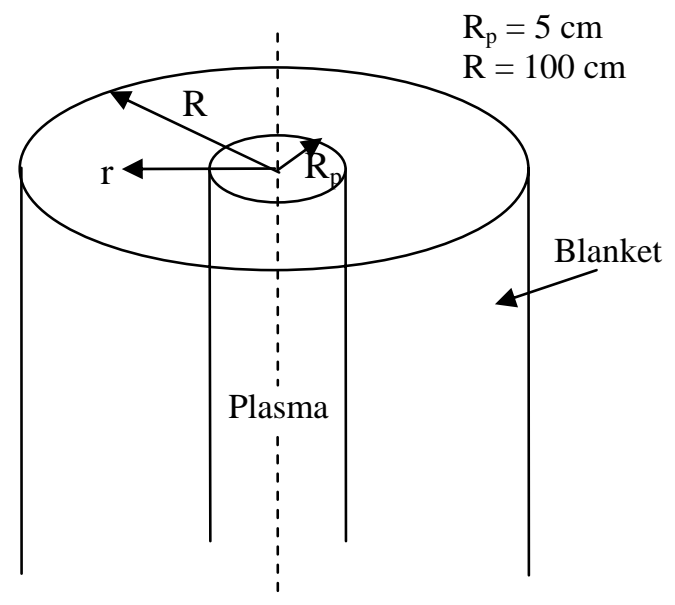

Figure 1. Fusion plasma and blanket. question are averaged over the neutron spectrum of integrated flux. In steady state, Eq. (1) yields

$$
N^{33}=\frac{\sigma_{\gamma}}{\sigma_{f}} N^{32}=\xi N^{32}
$$

with $\xi=0.1$ for fast neutrons. ${ }^{2}$ We note therefore that in steady state operation, the number density of $\mathrm{U}^{233}$ is 0.1 of that of thorium. In order to calculate the power density in the blanket, the neutron flux must be obtained, and we get it from the neutron balance equation, namely

$$
\frac{d n}{d t}=D \nabla^{2} \varphi-\Sigma_{a t} \varphi-\Sigma_{f} \varphi+S
$$

where $\Sigma_{a t}=\Sigma_{a 32}+\Sigma_{a 33}$. In other words, the absorption cross section is the combined one for the thorium and the uranium. According to Ref. 1,

$$
\Sigma_{a 32} \approx \Sigma_{a 33}
$$

which allows us to rewrite Eq. (3) in steady state as

American Institute of Aeronautics and Astronautics 


$$
D \nabla^{2} \varphi-\left(2 \Sigma_{a 33}+\Sigma_{f}\right) \varphi=0
$$

with the source term treated as a boundary condition. If we now let

$$
\alpha^{2}=\frac{2 \Sigma_{a 33}+\Sigma_{f}}{D}=\frac{1}{L^{2}}
$$

where $L$ is the diffusion length. Then Eq. (5) can be expressed as

$$
\nabla^{2} \varphi-\alpha^{2} \varphi=0
$$

The solution to the above equation in cylindrical coordinates is given by

$$
\varphi(r)=A I_{0}(\alpha r)+B K_{0}(\alpha r)
$$

where $I_{0}$ and $K_{0}$ are the modified Bessel functions. The boundary conditions of interest are:

$$
\begin{aligned}
& \varphi(R)=0 \\
& \left.J\right|_{R_{p}}=-\left.D \frac{d \varphi}{d r}\right|_{R_{p}}=S\left(2 \pi R_{p}\right)
\end{aligned}
$$

implying that the flux vanishes at the outer boundary of the blanket in the first condition, while maintaining the continuity of the neutron current at the plasma-blanket interface. It is clear from the first of Eq. (9) that $A=0$ assuming that $R$ is sufficiently large to satisfy this condition. Noting that

$$
\begin{aligned}
& K_{0}(z) \underset{z \rightarrow 0}{\longrightarrow}-\ln z \\
& \frac{d K_{0}(z)}{d z}=-K_{1}(z) \\
& K_{1}(z) \underset{z \rightarrow 0}{\longrightarrow} \frac{1}{2}\left(\frac{2}{z}\right)
\end{aligned}
$$

we see that satisfying the second condition of Eq. (9) leads to

$$
B=\frac{2 \pi R_{p}^{2}}{D} S
$$

with the final form of the neutron flux given by

$$
\varphi(r)=\frac{2 \pi R_{p}^{2}}{D} S K_{0}\left(\frac{r}{L}\right)
$$

We proceed now to calculate the diffusion coefficient $D$ and the diffusion length $L$ in order to evaluate the neutron flux in the various regions of the blanket. Noting that

$$
D=\frac{1}{3 \Sigma_{t r}}, \quad L^{2}=\frac{D}{2 \Sigma_{a 33}+\Sigma_{f}}
$$


where the transport cross section $\Sigma_{t r}$ is the sum of absorption and scattering cross sections, we assume that these quantities are primarily those of $\mathrm{U}^{233}$ for which we find that $D \approx 24 \mathrm{~cm}$ and $L \approx 39 \mathrm{~cm}$. The source term $S$ comes from the standard fusion expression, namely

$$
S=\frac{n_{p}^{2}}{4}\langle\sigma v\rangle
$$

where $n_{p}$ is the plasma density in the GDM, which we take to be $10^{-16} \mathrm{~cm}^{-3}$, and $\langle\sigma v\rangle$ for DT at $10 \mathrm{keV}$ temperature is about $10^{16} \mathrm{~cm}^{3} \mathrm{sec}^{-1}$, giving us a value of $S \approx 10^{16} / 4$ neutrons $/ \mathrm{cm}^{3} / \mathrm{sec}$. For a representative value of the flux, and correspondingly the power density in the blanket, we choose a point midway in the blanket, i.e. $r=50 \mathrm{~cm}$, and substitute in Eq. (12) using Eqs. (13) and (14) to obtain $\varphi(r=50) \approx 4.75 \times 10^{15}$ neutrons $/ \mathrm{cm}^{2} / \mathrm{sec}$. Using this value of the flux, and assuming $200 \mathrm{MeV}$ energy produced per fission, we find that an approximately mean value of the power density in the blanket is $785 \mathrm{Watts} / \mathrm{cm}^{3}$. If we compare this with the fusion power density produced in the GDM system, namely about $11 \mathrm{Watts} / \mathrm{cm}^{3}$, we see that a power amplification of about 71 is obtained by using the breeding approach addressed above. One can view this hybrid system as equivalent to a fusion power system with a Q-value of 71, often deemed as totally unrealistic in current or future fusion devices. Moreover, since criticality is completely avoided in this approach, the usual risks and hazards associated with it are totally circumvented.

\section{Conclusion}

We have demonstrated in this paper that a GDM bi-modal fusion propulsion system operating at $\mathrm{Q}>\sim 1$ can be effectively used as a surface power device producing large amounts of power when a "breeding" blanket is used. The fertile material is thorium-232, which breeds $\mathrm{U}^{233}$ with fast neutrons, and in steady state operation, the fusion neutrons cause the breeding and simultaneously induce fission in $\mathrm{U}^{233}$ to produce power. This approach is devoid of criticality and the risks and hazards associated with it.

\section{References}

\footnotetext{
${ }^{1}$ Kammash, T., and Tang, R., “Antiproton-Driven Fusion Propulsion System for OTV Applications,” 43 ${ }^{\text {rd }}$ Joint Propulsion Conference, Cincinnati, OH, 2007. AIAA-2007-5610.

${ }^{2}$ Rubbia, C., Proceedings of the International Conference on Accelerator-Driven Transmutation Technologies and Applications, AIP Conf. Proc. No. 346, Las Vegas, 1994.
} 